\title{
MATHIAS ABSOLUTENESS AND THE RAMSEY PROPERTY
}

\author{
LORENZ HALBEISEN AND HAIM JUDAH
}

\begin{abstract}
In this article we give a forcing characterization for the Ramsey property of $\Sigma_{2}^{1}$-sets of reals. This research was motivated by the well-known forcing characterizations for Lebesgue measurability and the Baire property of $\Sigma_{2}^{1}$-sets of reals. Further we will show the relationship between higher degrees of forcing absoluteness and the Ramsey property of projective sets of reals.
\end{abstract}

§1. Notations and definitions. Most of our set-theoretical notations and notations of forcings are standard and can be found in [9] or [16]. An exception is, that we will write $A^{B}$ for the set of all functions from $B$ to $A$, instead of ${ }^{B} A$ because we never use ordinal arithmetic. $A^{<\omega}$ is the set of all partial functions $f$ from $\omega$ to $A$, such that the cardinality of $\operatorname{dom}(f)$ is finite.

First we will give the definitions of the sets we will consider as the real numbers.

Let $[x]^{\kappa}:=\{y \subseteq x:|y|=\kappa\}$ and $[x]^{<\kappa}:=\{y \subseteq x:|y|<\kappa\}$, where $|y|$ denotes the cardinality of $y$. For $x \in[\omega]^{\omega}$, we will consider $[x]^{<\omega}$ as the set of strictly increasing, finite sequences in $x$ and $[x]^{\omega}$ as the set of strictly increasing, infinite sequences in $x$. For $x \in[\omega]^{\omega}$ and $n \in \omega$ let $x(n)$ be such that $x(n) \in x$ and $|x(n) \cap x|=n$.

We can consider $[\omega]^{\omega}$ also as a set of infinite 0 -1-sequences

$$
\begin{aligned}
{[\omega]^{\omega} } & \longrightarrow 2^{\omega} \\
x & \longmapsto
\end{aligned} \text { such that } f(n)=1 \text { iff } n \in x,
$$

or as the infinite sequences in $\omega$

$$
\begin{aligned}
& {[\omega]^{\omega \prime} \longrightarrow \omega^{\omega}} \\
& x \quad \longmapsto\left\langle a_{n}: n \in \omega\right\rangle \text { such that: } a_{0}:=x(0) \text { and } \\
& a_{n+1}:=x(n+1)-x(n)-1 \text {. }
\end{aligned}
$$

Note that these two mappings are bijective.

1.1. The Baire space. The Baire space is the space $\omega^{\omega}$ of all infinite sequences of natural numbers, $\left\langle a_{n}: n \in \omega\right\rangle$, with the following topology: For every finite sequence $s=\left\langle a_{k}: k<n\right\rangle$, let

$$
U_{s}:=\left\{f \in \omega^{\omega}: s \subset f\right\}=\left\{\left\langle c_{k}: k \in \omega\right\rangle: \forall k<n\left(c_{k}=a_{k}\right)\right\} .
$$

Received January 31, 1994; revised November 10, 1994.

The research for this paper was partially supported by the Emmy Nöther Institute in mathematics of Bar-Ilan and the Minerva foundation, Germany. 
The sets $U_{s}\left(s \in \omega^{<\omega}\right)$ form a basis for the topology of $\omega^{\omega}$. Note that each $U_{s}$ is also closed. The Baire space is homeomorphic to the space of all irrational numbers in $[0,1]$ with the topology of the real line (cf. [9] p. 36).

Because the mapping given above between $[\omega]^{\omega}$ and $\omega^{(\omega)}$ is bijective, we can endow $[\omega]^{\omega}$ with the induced topology and will not distinguish between the two spaces $[\omega]^{\omega}$ and $\omega^{\omega}$. The same holds for the sets $[\omega]^{\omega}$ and $2^{\omega}$.

1.2. Three properties of sets of reals. Let us work in the topological space $[\omega]^{(\omega)}$.

A set $R \subseteq[\omega]^{\omega}$ is rare (or nowhere dense) if the complement of $R$ contains a dense open set and a set $M \subseteq[\omega]^{\omega}$ is meager (or of first category) if $M$ is the union of countably many rare sets. A nonmeager set is also called a set of second category. A set $A \subseteq[\omega]^{\omega}$ has the Baire property if there exists an open set $G \subseteq[\omega]^{\omega}$ such that the symmetric difference $A \Delta G=(A \backslash G) \cup(G \backslash A)$ is meager.

A set $N \subseteq[\omega]^{\omega}$ is null if $N$ considered as a set of reals has Lebesgue measure zero. A set $A \subseteq[\omega]^{\omega}$ is Lebesgue measurable if there is a Borel set $B$ such that the symmetric difference $A \Delta B$ is null.

A set $A \subseteq[\omega]^{(\nu)}$ has the Ramsey property (or is Ramsey) if $\exists x \in[\omega]^{\omega \prime}\left([x]^{(\prime)} \subseteq\right.$ $\left.A \vee[x]^{\omega} \cap A=\emptyset\right)$. If there exists an $x$ such that $[x]^{\omega} \cap A=\emptyset$ we call $A$ a Ramsey。 set and if $[x]^{\omega} \subseteq A$ we call $A$ a co-Ramsey。 set. Note that $A$ can also be both. A set $A \subseteq[\omega]^{\omega}$ is called uniformly Ramsey ${ }_{\circ}$ if, for each $x \in[\omega]^{\omega}$ there is a $y \in[x]^{\omega}$ such that $[y]^{\omega} \cap A=\emptyset$.

1.3. The hierarchy of projective sets. We always consider the boldface $\Sigma_{n}^{1}$ hierarchy (see [9] p. 510). A $\Sigma_{1}^{1}$-set is the projection of a closed set. The $\Sigma_{1}^{1}$-sets are also called analytic sets. The $\Pi_{1}^{1}$-sets are the complements of the analytic sets. A $\Sigma_{n+1}^{1}$-set is the projection of a $\Pi_{n}^{1}$-set and the $\Pi_{n+1}^{1}$-sets are the complements of the $\Sigma_{n+1}^{1}$-sets. A set is $\Delta_{n}^{1}$ if it is $\Sigma_{n}^{1}$ and $\Pi_{n}^{1}$. For the normal form of the formulas representing projective sets and relations cf. [9] Section 40. Further we will consider a $\Sigma_{n}^{1}$-relation without free variables as a $\Sigma_{n}^{1}$-sentence.

If all $\Sigma_{n}^{1}$-sets with parameters in $V \cap W$ are Ramsey, (are Lebesgue measurable, have the Baire property, respectively), with respect to $\mathrm{V}$, we will write $V \models \Sigma_{n}^{1}(\mathscr{R})_{W}$ $\left(V \models \Sigma_{n}^{1}(\mathscr{L})_{W}, V \models \Sigma_{n}^{1}(\mathscr{B})_{W}\right.$, respectively). If $V=W$, then we do not write the index $W$. The notations $\Delta_{n}^{1}(\mathscr{R})_{W}, \Delta_{n}^{1}(\mathscr{L})_{W}, \Delta_{n}^{1}(\mathscr{B})_{W}, \Pi_{n}^{1}(\mathscr{R})_{W}, \Pi_{n}^{1}(\mathscr{L})_{W}$ and $\Pi_{n}^{1}(\mathscr{B})_{W}$ are similar. Note that because the three properties are closed under complements, the statements $\Sigma_{n}^{1}(\mathscr{R}), \Sigma_{n}^{1}(\mathscr{L})$ and $\Sigma_{n}^{1}(\mathscr{B})$ are equivalent to $\Pi_{n}^{1}(\mathscr{R})$, $\Pi_{n}^{1}(\mathscr{L})$ and $\Pi_{n}^{1}(\mathscr{B})$, respectively.

1.4. Filters and families on $\omega$. $\mathscr{F} \subseteq[\omega]^{\omega}$ is a Ramsey family if for all $\pi \in 2^{[\omega]^{2}}$ there is an $h \in \mathscr{F}$ such that $\left.\pi\right|_{[h]^{2}}$ is constant.

$\mathscr{F} \subseteq[\omega]^{\omega}$ is a dominating family if for all $x \in[\omega]^{\omega}$ there is a $d \in \mathscr{F}$ and a natural number $n \in \omega$ such that for all $k \geq n: d(k) \geq x(k)$.

$\mathscr{F} \subseteq[\omega]^{\omega}$ is dominated by the real $d$ if for all $f \in \mathscr{F}$ there is a natural number $n \in \omega$ such that for all $k \geq n: d(k) \geq f(k)$. (In this case we call $d$ a dominating real with respect to $\mathscr{F}$.)

$\mathscr{F} \subseteq[\omega]^{\leq \omega}$ is a filter (on $\left.\omega\right)$ if $\omega \in \mathscr{F}$ and for all $x, y \in[\omega]^{\leq \omega}$ : if $x, y \in \mathscr{F}$ then $x \cap y \in \mathscr{F}$ and if $x \in \mathscr{F}, x \subseteq y$ then $y \in \mathscr{F}$.

A filter $\mathscr{F}$ is proper if $\emptyset \notin \mathscr{F}$.

A filter $\mathscr{F}$ is an ultrafilter if it is proper and for every $x \in[\omega]^{\leq(1)}$, either $x \in \mathscr{F}$ or $\omega \backslash x \in \mathscr{F}$. 
The filter $\mathscr{F}=\left\{x \in[\omega]^{\omega}:|\omega \backslash x|<\omega\right\}$ is called the Fréchet filter.

A Ramsey ultrafilter is a Ramsey family which is also an ultrafilter. We consider only filters which are proper and contain the Fréchet filter.

1.5. Some notions of forcing. We recall the definition of the following seven notions of forcing.

(i) The Amoeba (measure) forcing $\boldsymbol{A}$ :

$$
\begin{aligned}
p \in A \Leftrightarrow p \subseteq & 2^{\omega} \text { is a perfect tree } \wedge \mu(p)>\frac{1}{2}, \\
& p \leq q \Leftrightarrow p \subseteq q .
\end{aligned}
$$

(ii) The Random forcing $\boldsymbol{B}$ :

$$
\begin{aligned}
p \in B \Leftrightarrow p \subseteq & 2^{\omega} \text { is a perfect tree } \wedge \mu(p)>0, \\
& p \leq q \Leftrightarrow p \subseteq q .
\end{aligned}
$$

(iii) The Cohen forcing $C$ :

$$
\begin{gathered}
p \in C \Leftrightarrow p \in 2^{<\omega}, \\
p \leq q \Leftrightarrow p \text { extends } q .
\end{gathered}
$$

(iv) The Hechler forcing $D$ :

$$
\begin{gathered}
\langle n, f\rangle \in D \Leftrightarrow n \in \omega \wedge f \in \omega^{\omega} \\
\langle n, f\rangle \leq\langle m, g\rangle \Leftrightarrow n \geq\left. m \wedge f\right|_{m}=\left.g\right|_{m} \wedge \forall k(f(k) \geq g(k)) .
\end{gathered}
$$

(v) The Mathias forcing $M$ :

$$
\begin{gathered}
\langle s, S\rangle \in M \Leftrightarrow s \in[\omega]^{<\omega} \wedge S \in[\omega]^{\omega} \wedge \max (\operatorname{range}(s))<\min (S), \\
\langle s, S\rangle \leq\langle t, T\rangle \Leftrightarrow s \text { extends } t \wedge S \subseteq T \wedge \forall i \in \operatorname{dom}(s) \backslash \operatorname{dom}(t)(s(i) \in T) .
\end{gathered}
$$

(vi) The forcing notion $\boldsymbol{P}(D)$ for an ultrafilter $D$ :

$$
\begin{gathered}
p_{s} \in \boldsymbol{P}(D) \Leftrightarrow \quad p_{s} \subseteq[\omega]^{<\omega} \text { is a tree and there is an } s \in p_{s} \text { such that } \\
\forall t \in p_{s}\left((s \subseteq t \vee t \subseteq s) \wedge\left(s \subseteq t \rightarrow\left\{n: t \sim n \in p_{s}\right\} \in D\right)\right), \\
p_{s} \leq q_{t} \Leftrightarrow p_{s} \subseteq q_{t} .
\end{gathered}
$$

(vii) The forcing notion $\boldsymbol{P}_{D}$ for an ultrafilter $D$ :

$$
\begin{aligned}
\langle s, a\rangle \in \boldsymbol{P}_{D} & \Leftrightarrow s \in[\omega]^{<\omega} \wedge a \in[\omega]^{\omega} \wedge a \in D \wedge \max (\operatorname{range}(s))<\min (a), \\
\langle s, a\rangle \leq\langle t, b\rangle & \Leftrightarrow s \text { extends } t \wedge a \subseteq b \wedge \forall i \in(\operatorname{dom}(s) \backslash \operatorname{dom}(t))(s(i) \in b) .
\end{aligned}
$$

In the forcing notions (v), (vi) and (vii) we call $s$ the stem of the condition $\langle s, S\rangle$, $p_{s}$ and $\langle s, a\rangle$, respectively. A generic object over one of these seven forcing notions can be considered as a generic real and we will handle the generic reals like the corresponding generic objects. For example if $G_{M}$ is Mathias generic and $p \in G_{M}$ (for a Mathias condition $p$ ), then we write $p \in m$ (for $m$ Mathias generic real) and if $p$ has empty stem $(p=\langle\emptyset, S\rangle)$, we also write $m \subseteq p$. Note that the conditions of these seven forcing notions can also be considered as reals, (and the meaning of $r_{1} \leq r_{2}$ is clear). Let $p, q$ be Mathias conditions, then we write $p \leq{ }^{0} q$ to say that $p$ and $q$ have the same stem and $p \leq q$.

Names in the forcing language are denoted with a " $\sim$ " over the letter. Canonical names for generic objects are usually denoted by boldface letters and canonical names for objects in the ground model we denote with a " $\vee$ " over the letter. 
1.6. Forcing-absoluteness. Let $\boldsymbol{P}$ be a notion of forcing. We define

$$
V^{P} \models \Phi \Longleftrightarrow V \models " 1 \Vdash_{P} \Phi "
$$

where $\Phi$ is a formula with parameters in $V$ and $\mathbf{1}$ is the weakest condition of $\boldsymbol{P}$.

Now we say $V$ is $\Sigma_{n}^{1}-\boldsymbol{P}$-absolute if for all $\Sigma_{n}^{1}$-sentences $\varphi$ with parameters in $V$,

$$
V^{P} \models \varphi \text { iff } V \models \varphi \text {. }
$$

Or equivalently, if for all $\boldsymbol{P}$-generic objects $G_{\boldsymbol{P}}$ over $V$ :

$$
V\left[G_{P}\right] \models \varphi \text { iff } V \models \varphi .
$$

§2. Introduction. In this section we give a list of results. Some of them are well-known, others gave the motivation to this work.

2.1. Characterizations with generic reals. Because the canonical well-ordering of constructible reals is $\Delta_{2}^{1}$ (cf. [9] Theorem 97), Gödel's constructible universe $L$ is neither a model for $\Delta_{2}^{1}(\mathscr{B})$ nor $\Delta_{2}^{1}(\mathscr{L})$ nor $\Delta_{2}^{1}(\mathscr{R})$. Hence, a model $V$ of set theory in which one of these properties holds, has to be larger than $L$. In fact, $V$ has even to contain even some reals which are generic over $L$.

THEOREM 2.1 .

(i) $V \models \Delta_{2}^{\mathrm{1}}(\mathscr{B})$ if and only if for all reals $r \in V$ the set of reals in $V$ which are Cohen over $L[r]$ is not empty.

(ii) $V \models \Delta_{2}^{1}(\mathscr{L})$ if and only if for all reals $r \in V$ the set of reals in $V$ which are random over $L[r]$ is not empty.

(iii) $V \models \Delta_{2}^{1}(\mathscr{R})$ if and only if for all reals $r \in V$ the set of reals in $V$ which are Ramsey over $L[r]$ is not empty.

Proof. All three results were proved in [14].

We also have a similar characterization for $\Sigma_{2}^{1}$-sets.

THEOREM 2.2.

(i) $V \models \Sigma_{2}^{1}(\mathscr{B})$ if and only if for all reals $r \in V$ the set of reals in $V$ which are Cohen over $L[r]$ is co-meager.

(ii) $V \models \Sigma_{2}^{1}(\mathscr{L})$ if and only if for all reals $r \in V$ the set of reals in $V$ which are random over $L[r]$ has measure 1.

(iii) $V \models \Sigma_{2}^{1}(\mathscr{R})$ if and only if for all reals $r \in V$ the set of reals in $V$ which are Ramsey over $L[r]$ is co-Ramsey。.

Proof. A proof can be found in [1]. For the third result see also [14].

2.2. Characterizations with forcing absoluteness. For the $\Sigma_{2}^{1}$-sets we also find a characterization with forcing absoluteness.

THEOREM 2.3.

(i) $V \models \Sigma_{2}^{1}(\mathscr{B})$ if and only if $V$ is $\Sigma_{3}^{1}$-Hechler-absolute.

(ii) $V \equiv \Sigma_{2}^{1}(\mathscr{L})$ if and only if $V$ is $\Sigma_{2}^{1}$-Amoeba-absolute.

(iii) $V \models \Sigma_{2}^{1}(\mathscr{R})$ if and only if $V$ is $\Sigma_{3}^{1}$-Mathias-absolute.

Proof. The first two results were proved in [13] and [12]. A proof of the last one will be given in this work, Theorem 4.1. 
For higher levels in the projective hierarchy, we lose the forcing characterization with Mathias forcing for the Ramsey property. We will show in Theorem 5.3 that

$$
\Sigma_{4}^{1} \text {-Mathias-absoluteness } \Rightarrow \Sigma_{3}^{1}(\mathscr{R})
$$

but (Theorem 5.2)

$$
\Delta_{3}^{1}(\mathscr{R}) \nRightarrow \Sigma_{4}^{1} \text {-Mathias-absoluteness. }
$$

The reason for this is, that if $V$ is $\Sigma_{4}^{1}$-Mathias-absolute, then $\omega_{1}^{V}$ is inaccessible in $L$. On the other hand we can build a model in which $\Delta_{3}^{1}(\mathscr{R})$ holds without using inaccessible cardinals. We will show further (Corollary 6.1) that

$$
\Sigma_{5}^{1} \text {-Mathias-absoluteness } \Rightarrow \Delta_{4}^{1}(\mathscr{R}),
$$

and moreover (Corollary 6.5)

$$
\Sigma_{6}^{1} \text {-Mathias-absoluteness } \Rightarrow \Delta_{5}^{1}(\mathscr{R}) \text {. }
$$

§3. The Ramsey property and Mathias forcing.

\subsection{Basic facts about the Ramsey property.}

FACT 3.1. If $A \subseteq[\omega]^{\omega}$ is Ramsey and $C \subseteq[\omega]^{\omega}$ is uniformly Ramsey。 (e.g., countable), then both, $A \cup C$ and $A \backslash C$ are Ramsey.

Proof. To see that $A \cup C$ is Ramsey, first note that if there is an $x \in[\omega]^{\omega}$ such that $[x]^{\omega} \subseteq A$, we are done. Otherwise, pick $x$ such that $[x]^{\omega} \cap A=\emptyset$ and pick $y \in[x]^{\omega}$ such that $[y]^{\omega} \cap C=\emptyset$. Now $[y]^{\omega} \cap(A \cup C)=\emptyset$.

To see that $A \backslash C$ is Ramsey, again note that if there is an $x$ such that $[x]^{\omega} \cap A=\emptyset$, we are done. Otherwise, pick $x$ such that $[x]^{\omega} \subseteq A$. Now there is a $y \in[x]^{\omega}$ such that $[y]^{\omega} \cap C=\emptyset$ and $[y]^{\omega} \subseteq(A \backslash C)$.

FACT 3.2. The axiom of choice implies that there are sets without the Ramsey property.

Proof. Define on $[\omega]^{\omega \nu}$ an equivalence-relation as follows:

$$
x \sim y \text { iff }|x \Delta y| \text { is finite. }
$$

Now choose from each equivalence class $x^{\sim}$ an element $c_{x}$. Further define:

$$
f(x):= \begin{cases}1 & \text { if }\left|x \Delta c_{x}\right| \text { is odd } \\ 0 & \text { otherwise. }\end{cases}
$$

Then the set $\{x: f(x)=1\}$ is evidently not Ramsey.

The first example of a set which does not have the Ramsey property is given in [7]. A lot of other examples can be found in [4] and [5].

FACT 3.3. Analytic sets (these are the $\Sigma_{1}^{1}$-sets) are Ramsey.

Proof. A proof can be found in [6] and [18]. 
3.2. The forcing notions $\boldsymbol{P}(D), \boldsymbol{P}_{D}$ and $\boldsymbol{M}$. (Compare also with [17]).

Let $\mathscr{J}=[\omega]^{<\omega}$ be the ideal of finite sets and let $\langle\mathscr{P}(\omega) / \mathscr{J}, \leq\rangle=: \boldsymbol{U}$ be the partial order defined as follows:

$$
\begin{gathered}
p \in U \Leftrightarrow p \in[\omega]^{\omega}, \\
p \leq q \Leftrightarrow p \backslash q \in \mathscr{J} \text { (this is } p \subseteq^{*} q \text { ). }
\end{gathered}
$$

FACT 3.4. Let $D$ be $U$-generic over $V$, then $D$ is a Ramsey ultrafilter in $V[D]$.

Proof. First note that $U$ is $\aleph_{0}$-closed, hence adds no new reals to $V$, (cf. [9] Lemma 19.6). Let $\pi \in 2^{[\omega]^{2}}$, then by the Ramsey Theorem (cf. [9] Lemma 29.1) for each $p \in[\omega]^{\omega}$ there exists a $q \subseteq^{*} p$ such that $\pi$ is constant on $[q]^{2}$. Therefore, $H_{\pi}:=\left\{q \in[\omega]^{\omega}:\left.\pi\right|_{[q]^{2}}\right.$ is constant $\}$ is dense in $\boldsymbol{U}$, hence $H_{\pi} \cap D \neq \emptyset$.

LEMMA 3.5. Let $\tilde{D}$ be the canonical $U$-name for the $U$-generic object, then

$$
\boldsymbol{U} * \boldsymbol{P}_{\tilde{D}} \approx \boldsymbol{M} .
$$

ProOF.

$$
\begin{aligned}
\boldsymbol{U} * \boldsymbol{P}_{\tilde{D}} & =\left\{\langle p,\langle\tilde{s}, \tilde{a}\rangle\rangle: p \in \boldsymbol{U} \wedge p \vdash_{U}\langle\tilde{s}, \tilde{a}\rangle \in \boldsymbol{P}_{\tilde{D}}\right\} \\
& =\left\{\langle p,\langle\tilde{s}, \tilde{a}\rangle\rangle: p \in[\omega]^{\omega} \wedge p \vdash_{U}(\tilde{a} \in \tilde{D} \wedge \max (\operatorname{range}(\tilde{s}))<\min (\tilde{a}))\right\} .
\end{aligned}
$$

Now the embedding

$$
h: \begin{array}{rll}
\boldsymbol{M} & \longrightarrow \boldsymbol{U} * \boldsymbol{P}_{\tilde{D}} \\
\langle s, a\rangle & \longmapsto\langle a,\langle\tilde{s}, \tilde{a}\rangle\rangle
\end{array}
$$

is a dense embedding (see [8] Definition 0.8):

(1) It is easy to see, that $h$ preserves the order relation $\leq$.

(2) Let $\langle p,\langle\tilde{s}, \tilde{a}\rangle\rangle \in \boldsymbol{U} * \boldsymbol{P}_{\tilde{D}}$. Because $\boldsymbol{U}$ is $\aleph_{0}$-closed, there is a condition $q \leq p$ and $s \in[\omega]^{<\omega}, a \in[\omega]^{\omega}$ such that $q \vdash_{U} \check{s}=\tilde{s} \wedge \check{a}=\tilde{a}$. It is obvious that $\langle q,\langle\check{s}, \breve{a}\rangle\rangle \in \boldsymbol{U} * \boldsymbol{P}_{\tilde{D}}$ is stronger than $\langle p,\langle\tilde{s}, \tilde{a}\rangle\rangle$. Now let $b:=q \cap a$, then $h(\langle s, b\rangle) \leq\langle p,\langle\tilde{s}, \tilde{a}\rangle\rangle$.

Lemma 3.6. $\boldsymbol{P}_{D} \approx \boldsymbol{P}(D)$ if and only if $D$ is a Ramsey ultrafilter.

Proof. See [14] Theorem 1.20.

LEMMA 3.7. The Mathias forcing $\boldsymbol{M}$ is flexible.

Proof AND DEFINITION.For the notation see [9] p. 153 and [16] p. 224.

A set $T \subseteq \omega^{<\omega}$ is called a Laver-tree if

$T$ is a tree and $\exists \tau \in T \forall \sigma \in T(\sigma \subseteq \tau \vee(\tau \subseteq \sigma \wedge|\{n: \sigma \sim n \in T\}|=\omega))$.

(We call $\tau$ the stem of $T$. For $\sigma \in T$ we let $\operatorname{succ}_{T}(\sigma):=\left\{n: \sigma^{\frown} n \in T\right\}$, (the successors of $\sigma$ in $T$ ) and $T_{\rho}:=\{\sigma \in T: \sigma \subseteq \rho \wedge \rho \subseteq \sigma\}$.)

A Laver-tree $T$ is uniform if there exists $u_{T} \in[\omega]^{\omega}$ such that $\forall \sigma \supseteq \operatorname{stem}(T)(\{n$ : $\left.\sigma^{\frown} n \in T\right\}=u_{T} \backslash(\max (\sigma)+1)$.

For a Laver-tree $T$, we say $A \subseteq T$ is a front if $\sigma \neq \tau$ in $A$ implies $\sigma \not \tau$ and for all $f \in[T]$ there is an $n \in \omega$ such that $\left.f\right|_{n} \in A$. 
The meaning of $p \leq \llbracket \Phi \rrbracket$ and $p \cap \llbracket \Phi \rrbracket$ are $U_{p} \subseteq \llbracket \Phi \rrbracket$ and $U_{p} \cap \llbracket \Phi \rrbracket$, respectively.

(1) We say a forcing notion $\boldsymbol{P}$ is Laver-like if there is a $\boldsymbol{P}$-name $\tilde{\boldsymbol{r}}$ for a dominating real such that

(i) the complete Boolean algebra generated by the family $\{\llbracket \tilde{r}(i)=n \rrbracket: i, n \in \omega\}$ equals r.o. $(\boldsymbol{P})$, and

(ii) for each condition $p \in \boldsymbol{P}$ there exists a Laver-tree $T \subseteq \omega^{\omega}$ so that

$$
\forall \sigma \in T\left(p\left(T_{\sigma}\right):=\prod_{n \in(\prime)} \sum_{\tau \in T_{\sigma}}\left\{\left.p \cap \llbracket \tilde{r}\right|_{\lg (\tau)}=\tau \rrbracket: \lg (\tau)=n\right\} \in \text { r.o. }(\boldsymbol{P}) \backslash\{\boldsymbol{0}\}\right) .
$$

We express this by saying $p(T) \neq \emptyset$ where $p(T):=p\left(T_{\text {stem }(T)}\right)$.

$\boldsymbol{M}$ is Laver-like:

Proof. Let $\mathbf{m}$ be the canonical $\boldsymbol{M}$-name for the Mathias real, then $\mathbf{m}$ is dominating (cf. [10] Part I, Lemma 3.15) and further let $p=\langle s, S\rangle \in \boldsymbol{M}$ with $\lg (s)=n$ and $S=\{a(j): j \in \omega\}$. Then $U_{p}=\prod_{k \in n} \llbracket \mathbf{m}(\breve{k})=s(\check{k}) \rrbracket \cdot \prod_{i \in \omega} \sum_{j \in \omega} \llbracket \mathbf{m}(\check{n}+\check{i})=a(j) \rrbracket$, which gives a proof of (i).

For (ii) consider $T \subseteq \omega^{<(1)}$ defined as follows:

$$
\begin{aligned}
\sigma \in T \text { iff } & \sigma \text { strictly increasing and } \\
& \sigma \subseteq s \vee(s \subseteq \sigma \wedge \operatorname{range}(\sigma) \backslash \operatorname{range}(s) \subseteq S) .
\end{aligned}
$$

This $T$ has the desired property and is even a uniform Laver-tree.

(2) If $\tilde{r}$ is a $\boldsymbol{P}$-name that witnesses that $\boldsymbol{P}$ is Laver-like, we say that $\boldsymbol{P}$ has strong fusion if for countably many open dense sets $D_{n} \subseteq \boldsymbol{P}$ and for $p \in \boldsymbol{P}$, there is a Laver-tree $T$ such that $p(T) \neq \emptyset$ and for each $n$ :

$$
\left\{\sigma \in T:\left.p(T) \cap \llbracket \tilde{r}\right|_{\lg (\sigma)}=\sigma \rrbracket \in D_{n}\right\}
$$

contains a front.

$\boldsymbol{M}$ has strong fusion:

Proof. Let $D \subseteq \boldsymbol{M}$ be dense open and $p=\langle s, S\rangle$ an $\boldsymbol{M}$-condition. For each $\sigma$ such that $\sigma \subseteq s$ or $(s \subseteq \sigma \wedge \sigma \backslash s \subseteq S)$ we define the rank of $\sigma, \operatorname{rk}_{D}(\sigma)$ as follows:

$$
\begin{array}{rll}
\mathrm{rk}_{D}(\sigma)=0 \Leftrightarrow & \exists A \in[S]^{\omega}(\langle\sigma, A\rangle \in D), \\
\mathrm{rk}_{D}(\sigma)=\alpha \Leftrightarrow & \Rightarrow \exists \beta<\alpha\left(\mathrm{rk}_{D}(\sigma)=\beta\right) \text { and } \\
& \left|\left\{n: n \in S \wedge \mathrm{rk}_{D}(\sigma-n)<\alpha\right\}\right|=\omega .
\end{array}
$$

If $\operatorname{rk}_{D}(\sigma)$ is undefined, we put $\operatorname{rk}_{D}(\sigma)=\infty$.

Note that if $\sigma \in \operatorname{dom}\left(\operatorname{rk}_{D}\right)$, then $\operatorname{rk}_{D}(\sigma)<\infty$. Otherwise almost all successors (in $S$ ) of $\sigma$ have rank $=\infty$, hence the complement of $S_{0}:=\left\{n: n \in S \wedge \operatorname{rk}_{D}\left(\sigma^{-} n\right)=\right.$ $\infty\}$ with respect to $S$ is finite. Let $s_{n}:=\min \left(S_{n}\right)$, then the complement of

$$
S_{n+1}:=\left\{n: n \in S_{n} \wedge \operatorname{rk}_{D}\left(\widetilde{\sigma^{-}} \widetilde{S_{n}^{-}} n\right)=\infty \text { for all } \tau \in\left[\left\{s_{0}, \ldots, s_{n-1}\right\}\right]^{<n}\right\}
$$

with respect to $S_{n}$ is finite. Let $A:=\left\{s_{i}: i \in \omega\right\} \subseteq S$ and take $\left\langle\rho, A^{\prime}\right\rangle \leq\langle\sigma, A\rangle$ such that $\left\langle\rho, A^{\prime}\right\rangle \in D$. Then $\rho=\sigma^{\frown} \tau \frown s_{n}$ (for an $n$ ) and $A^{\prime} \in[A]^{\omega}$, hence $\operatorname{rk}_{D}(\rho)=\infty$, a contradiction. 
For two uniform Laver-trees $T$ and $T^{\prime}$, the expression $T \leq_{n} T^{\prime}$ means that the first $n$ elements of $u_{T}$ and $u_{T^{\prime}}$ are the same. Let $T_{0}$ be the uniform Laver-tree constructed in the proof of part (ii) above.

Define a uniform Laver-tree $T_{n+1}$ and the corresponding set $u_{T_{n+1}}$ recursively such that $T_{n+1} \leq_{n} T_{n}$ and if $\sigma \in T_{n+1}$ then one of the following is true:

$\max (\sigma) \leq u_{T_{n}}(n) \wedge \sigma \in T_{n}$

$\left(\operatorname{rk}_{D_{n}}(\sigma)=0 \wedge \forall k<\lg (\sigma)\left(\mathrm{rk}_{D_{n}}\left(\left.\sigma\right|_{k}\right)>0\right)\right) \rightarrow\left\langle\sigma, u_{T_{n+1}} \backslash(\max (\sigma)+1)\right\rangle \in D_{n}$

$\mathrm{rk}_{D_{n}}(\sigma)>0 \wedge \forall k \in u_{T_{n+1}} \backslash(\max (\sigma)+1)\left(\mathrm{rk}_{D_{n}}(\sigma)>\mathrm{rk}_{D_{n}}(\sigma-k)\right)$.

Now $T_{n+1}$ is a uniform Laver-tree and $T:=\bigcap_{n \in \omega} T_{n}$ is also uniform, $p(T) \neq \emptyset$ and $\left\{\sigma \in T:\left.p \cap \llbracket \mathbf{m}\right|_{\lg (\sigma)}=\sigma \rrbracket \in D_{n}\right\}$ contains a front, (consider $\mathrm{rk}_{D_{n}}$ ).

(3) A Laver-like $\boldsymbol{P}$ is closed under finite changes if given a $p \in \boldsymbol{P}$ and Laver trees $T$ and $T^{\prime}$ so that for all $\sigma \in T^{\prime}:\left|\operatorname{succ}_{T}(\sigma) \backslash \operatorname{succ}_{T^{\prime}}(\sigma)\right|<\omega$, if $p(T) \neq \emptyset$, then $p\left(T^{\prime}\right) \neq \emptyset$, too.

$\boldsymbol{M}$ is closed under finite changes:

Proof. Use a standard fusion argument.

(4) We call $\boldsymbol{P}$ a flexible forcing notion iff $\boldsymbol{P}$ is Laver-like, has strong fusion and is closed under finite changes.

Hence, the Mathias forcing $\boldsymbol{M}$ is flexible.

3.3. Essential theorems about $\Delta_{2}^{1}$-sets of reals. Now we will give the relationship between the Ramsey property and Mathias forcing.

FACTS 3.8 .

(1) [14] Theorem 1.7:

For every $\boldsymbol{P}(D)$-sentence $\Phi$ and for all $p \in \boldsymbol{P}(D)$ there exists a $q \in \boldsymbol{P}(D)$ such that $q \leq p, \operatorname{stem}(p)=\operatorname{stem}(q)$ and

$$
q \Vdash_{P(D)} \Phi \text { or } q \Vdash_{P(D)} \neg \Phi(q \text { decides } \Phi) \text {. }
$$

(This is known as pure decision.)

(2) [14] Theorem 1.14:

If $V \subseteq V^{\prime} \subseteq V^{\prime \prime}$ are models of ZFC and $D \in V$ is an ultrafilter and $x \in V^{\prime}$ is $\boldsymbol{P}(D)$-generic over $V$, then for every $y \in[x]^{\omega} \cap V^{\prime \prime}, y$ is $\boldsymbol{P}(D)$-generic over $V$, too.

(3) [14] Theorem 1.15:

If $D \in V$ and $g$ is $\boldsymbol{P}(D)$-generic over $V$, then

$$
V[g] \vDash \Sigma_{2}^{1}(\mathscr{R})_{V} .
$$

(4) [14] Theorem 1.16:

If $D \in V$, then

$$
r \in[\omega]^{\omega} \text { is } \boldsymbol{P}(D) \text {-generic over } V \text { if and only if }
$$

$\forall a \in D\left(r \subseteq \subseteq^{*} a\right)$ and $\forall \pi \in 2^{[\omega]^{2}} \cap V: \exists n \in \omega$ such that $\left.\pi\right|_{[r \backslash n]^{2}}$ is constant.

(5) [14] Theorem 2.7:

$$
V \models \Delta_{2}^{1}(\mathscr{R}) \text { if and only if } V \models \Sigma_{2}^{1}(\mathscr{R}) .
$$


(6) [14] Theorem 2.11:

For an $s \in[\omega]^{\omega}$ define $D_{s}:=\left\{a \in[\omega]^{\omega}: s \subseteq^{*} a\right\}$ (where $s \subseteq^{*} a$ means $|s \backslash a|<\omega)$ and $D^{s}:=D_{s} \cap L\left[D_{s}\right]$. If $D^{s}$ is an ultrafilter in $L\left[D^{s}\right]$ and $r \subseteq^{*} s$, then $D^{r}=D^{s}$ and we write $\boldsymbol{P}_{s}$ for the forcing notion $\boldsymbol{P}\left(D^{s}\right)$ in $L\left[D^{s}\right]$.

$$
V=\Delta_{2}^{1}(\mathscr{R})_{L[u]} \Leftrightarrow \forall r \in L[u] \exists s \in[r]^{\omega} \cap V\left(s \text { is } \boldsymbol{P}_{s} \text {-generic over } L[u]\left[D^{s}\right]\right) \text {. }
$$

\subsection{Some properties of Mathias forcing.}

FACTS 3.9. (1) Using the Fact 3.8 3. and the Lemmas 3.5 and 3.6 we see that if $m$ is Mathias over $V$, then $V[m] \models \Sigma_{2}^{1}(\mathscr{R})_{V}$. Thus, (with [16] Lemma 5.14 on p. 276) an $\omega_{1}$-iteration of Mathias forcing with countable support gives a model in which each $\Sigma_{2}^{1}$-set is Ramsey. (2) We call $r$ a Ramsey real over $V$ if and only if there exists a $D \in V$ such that:

(i) $D$ is an ultrafilter, $\forall a \in D\left(r \subseteq^{*} a\right)$ and

(ii) for all $\pi \in 2^{[\omega]^{2}}, \pi \in V$ there is an $n \in \omega$ such that $\left.\pi\right|_{[r \backslash n]^{2}}$ is constant.

(See also [14] Definition 1.17).

Now we see that if $s$ is $\boldsymbol{P}_{s}$-generic over $L[u]\left[D^{s}\right]$, then (by 3.84 .) it is Ramsey over $L[u]\left[D^{s}\right]$ and even a dominating real with respect to $L[u]\left[D^{s}\right]$.

Proof. To each real $r \in L[u]\left[D^{s}\right]$ consider the function $\pi_{r} \in 2^{[\omega]^{2}}$ (which also belongs to $L[u]\left[D^{s}\right]$ ) defined as follows:

$$
\pi_{r}(\{i, j\})=0 \Longleftrightarrow \exists k\left(r\left(2^{k}\right)<i, j \leq r\left(2^{k+1}\right)\right) .
$$

Because $s$ is $\boldsymbol{P}_{s}$-generic and by 3.84 . we have

$$
\exists n \in \omega\left(\left.\pi\right|_{[s \backslash n]^{2}}\right. \text { is constant). }
$$

Thus, because $s \backslash n$ is infinite, $\left.\pi\right|_{[s \backslash n]^{2}} \equiv 1$ and for $k \geq 2 n$ we get $s(k)>r(k)$, hence

$$
\forall r \in L[u]\left[D^{s}\right] \cap[\omega]^{\omega} \exists l \in \omega \forall k \geq l(s(k)>r(k))
$$

which says, that the reals of $L[u]\left[D^{s}\right]$ are dominated by $s$.

We close this section by mentioning two useful corollaries.

COROLlaRY 3.10. If $p$ is an $\boldsymbol{M}$-condition and $\tilde{x}$ is an $\boldsymbol{M}$-name for a real, then there exists an $M$-condition $q \leq 0$ and a real $\bar{x} \in V$ such that $V \models " q \Vdash_{M} \bar{x}=\tilde{x} "$.

PRoof. Let $\tilde{x}$ be an $\boldsymbol{M}$-name for a real. Each real can be considered as an infinite $0-1$-sequence, so $\tilde{x}$ is such that for all natural numbers $n$ :

$$
\tilde{x}(\check{n})=\check{1} \text { or } \tilde{x}(\check{n})=\check{0} \text {. }
$$

Take $p=\langle s, X\rangle$. Because Mathias forcing has pure decision (by the Lemmas 3.5, 3.6 and Fact 3.81 ., or by [2] Theorem 9.3) in $V$ there is a condition $\left\langle s, X_{0}\right\rangle$ such that $X_{0} \subseteq X$ which decides $\tilde{x}(\tilde{0})$. Let $a_{0}$ be the least member of $X_{0}$, then there are $Y, X_{1}$ such that $X_{0} \backslash\left\{a_{0}\right\} \supseteq Y \supseteq X_{1}$ and $\left\langle s-a_{0}, Y\right\rangle,\left\langle s, X_{1}\right\rangle$ both decide $\tilde{x}(\check{1})$. Let now $a_{1}$ be the least member of $X_{1}$. There are $Y_{1}, Y_{2}, Y_{3}, X_{2}$ such that $X_{1} \backslash\left\{a_{1}\right\} \supseteq Y_{1} \supseteq$ $\ldots \supseteq X_{2}$ and $\left\langle s \frown a_{0}^{\frown} a_{1}, Y_{1}\right\rangle,\left\langle s \frown a_{1}, Y_{2}\right\rangle,\left\langle s \frown a_{0}, Y_{3}\right\rangle,\left\langle s, X_{2}\right\rangle$ all decide $\tilde{x}(\tilde{2})$. Now let $a_{2}$ be the least member of $X_{2}$ and so on. Define $r:=\left\{a_{i}: i \in \omega\right\}$. We encode now $\tilde{x}$ by $\bar{x}:=\left\{s^{-} t: t \in[r]^{<\omega} \wedge\left\langle s^{-} t, r \backslash(\max (t)+1)\right\rangle \vdash_{M} \tilde{x}\left(\lg (t)^{\vee}\right)=\check{1}\right\}$. Then 
$\bar{x}$ is a real and if $m$ is a Mathias real over $V$ such that $\langle s, r\rangle \in m$ then $\tilde{x}[m]=\bar{x}[m]$, where $\bar{x}[m](n)=1$ if and only if $\left.m\right|_{n} \in \bar{x}$.

COROLlaRY 3.11. If $p$ is an $\boldsymbol{M}$-condition and $V \models " p \vdash_{M} \exists x \Phi(x)$ ", then there is an $\boldsymbol{M}$-condition $q \leq 0$ and an $\boldsymbol{M}$-name $\tilde{x}$ for a real such that $V \models " q \Vdash_{M} \Phi(\tilde{x})$ ".

Proof. We will follow the proofs of [2] Theorems 9.1 and 9.3.

Assume $p=\langle s, A\rangle \Vdash_{M} \exists x \Phi(x)$. First we prove that there is a $B \subseteq A$ such that if $\langle t, C\rangle \leq\langle s, B\rangle, \tilde{x}$ an $\boldsymbol{M}$-name and $\langle t, C\rangle \vdash_{M} \Phi(\tilde{x})$, then we find an $\boldsymbol{M}$-name $\tilde{y}$ such that $\langle t, B \backslash(\max (t)+1)\rangle \Vdash_{M} \Phi(\tilde{y})$. For this we construct a sequence $b_{0}<b_{1}<\ldots$ of elements of $A$ and a sequence $B_{0} \supseteq B_{1} \supseteq \ldots$ of subsets of $A$ such that for all $b \in B_{n+1}, b_{n}<b$. Let $B_{0}:=A$. Given $B_{n}$, let $s_{1}, s_{2}, \ldots, s_{k}$ enumerate all the subsets of $\left\{b_{i}: i<n\right\}$. Now construct a sequence $B_{n}^{0} \supseteq B_{n}^{1} \supseteq \ldots \supseteq B_{n}^{k}$ as follows. $B_{n}^{0}:=B_{n}$ and given $B_{n}^{i-1}$ let $B_{n}^{i} \subseteq B_{n}^{i-1}$ be such that for some $\boldsymbol{M}$-name $\tilde{x}$, $\left\langle s \cup s_{i}, B_{n}^{i}\right\rangle \vdash_{M} \Phi(\tilde{x})$, if it exists; otherwise let $B_{n}^{i}:=B_{n}^{i-1}$. Finally let $b_{n}:=\bigcap B_{n}^{k}$, $B_{n+1}:=B_{n}^{k} \backslash\left\{b_{n}\right\}$ and $B:=\left\{b_{n}: n \in \omega\right\}$. Suppose $\langle t, C\rangle \leq\langle s, B\rangle$ and we find an $M$-name $\tilde{x}$ such that $\langle t, C\rangle \Vdash_{M} \Phi(\tilde{x})$. Because there is an $n \in \omega$ such that $s_{l}:=t \backslash s \subseteq\left\{b_{i}: i<n\right\}$ we must have chosen $B_{n}^{l}$ so that for some $\boldsymbol{M}$-name $\tilde{y}$, $\left\langle s \cup s_{l}, B_{n}^{l}\right\rangle \vdash_{M} \Phi(\tilde{y})$. Now $B \backslash(\max (t)+1) \subseteq B_{n}^{l}$, hence $\langle t, B \backslash(\max (t)+1)\rangle \leq$ $\left\langle t, B_{n}^{l}\right\rangle \Vdash_{M} \Phi(\tilde{y})$ and we are done.

If $p, q$ are two $\boldsymbol{M}$-conditions, then $p \cap q$ denotes the weakest $\boldsymbol{M}$-condition which is stronger than $p$ and $q$, (if it exists). Let $\tilde{x}$ be an $\boldsymbol{M}$-name and $p$ an $\boldsymbol{M}$-condition, then $\tilde{x}(p)$ denotes the following name. $\langle\tilde{\sigma}, q\rangle \in \tilde{x}(p)$ if and only if there exists an $\boldsymbol{M}$-condition $q^{\prime}$ such that $\left\langle\tilde{\sigma}, q^{\prime}\right\rangle \in \tilde{x}$ and $q=p \cap q^{\prime}$. For two $\boldsymbol{M}$-names $\tilde{x}, \tilde{y}$ let $\tilde{x} \cup \tilde{y}:=\{\langle\tilde{\sigma}, p\rangle:\langle\tilde{\sigma}, p\rangle \in \tilde{x} \vee\langle\tilde{\sigma}, p\rangle \in \tilde{y}\}$.

Now we are prepared to prove the corollary. Given $p=\langle s, A\rangle \Vdash_{M} \exists x \Phi(x)$. Let $B \subseteq A$ be as above. We construct a sequence $b_{0}<b_{1}<\ldots$ of elements of $B$ and subsets $B_{0} \supseteq B_{1} \supseteq \ldots$ of $B$ by induction as follows. Let $B_{0}:=B$. Given $B_{n}$, find $B_{n+1}^{\prime} \subseteq B_{n}$ so that for all $s^{\prime} \subseteq\left\{b_{i}: i<n\right\}$ one of the following cases holds:

(1) For all $b \in B_{n+1}^{\prime}$ we find an $\boldsymbol{M}$-name $\tilde{x}$ (depending on $b$ ) such that $\left\langle s \cup s^{\prime} \cup\right.$ $\left.\{b\}, B_{n+1}^{\prime} \backslash(b+1)\right\rangle \vdash_{M} \Phi(\tilde{x})$.

(2) For no $b \in B_{n+1}^{\prime}$ we find an $\boldsymbol{M}$-name $\tilde{x}$ (which may depend on $b$ ) such that $\left\langle s \cup s^{\prime} \cup\{b\}, B_{n+1}^{\prime} \backslash(b+1)\right\rangle \vdash_{M} \Phi(\tilde{x})$.

Because of the choice of $B$, for each $n$ we find a $B_{n+1}^{\prime} \subseteq B_{n} \subseteq B$. Let $b_{n}:=\bigcap B_{n+1}^{\prime}$, $B_{n+1}:=B_{n+1}^{\prime} \backslash\left\{b_{n}\right\}$ and $A^{\prime}:=\left\{b_{n}: n \in \omega\right\}$. Suppose for $\langle t, C\rangle \leq\left\langle s, A^{\prime}\right\rangle$ we find an $\boldsymbol{M}$-name $\tilde{x}$, such that $\langle t, C\rangle \Vdash_{M} \Phi(\tilde{x})$. Let $|t|$ be minimal. If $|t|=|s|$ then $t=s$ and we find an $\boldsymbol{M}$-name $\tilde{y}$ such that $\left\langle s, A^{\prime}\right\rangle \Vdash_{M} \Phi(\tilde{y})$. If $|t|>|s|$ then $\max (t)=b_{n}$ for some $n$ and at stage $n$, the first case held for some $s^{\prime}=t \backslash\left(s \cup\left\{b_{n}\right\}\right)$. Now for each $b_{i} \in A^{\prime}(i \geq n)$ take an $\boldsymbol{M}$-name $\tilde{x}_{i}$ such that $\left\langle s \cup s^{\prime} \cup\left\{b_{i}\right\}, A^{\prime} \backslash\left(b_{i}+1\right)\right\rangle \Vdash_{M} \boldsymbol{\Phi}\left(\tilde{x}_{i}\right)$. Further let $\tilde{y}:=\bigcup\left\{\tilde{x}_{i}\left(p_{i}\right): i \geq n \wedge p_{i}=\left\langle s \cup s^{\prime} \cup\left\{b_{i}\right\}, A^{\prime} \backslash\left(b_{i}+1\right)\right\rangle\right\}$. Then we have $\left\langle s \cup s^{\prime}, A^{\prime} \backslash\left(\max \left(s^{\prime}\right)+1\right)\right\rangle \Vdash_{M} \Phi(\tilde{y})$, which is a contradiction to the minimality of $|t|$.

$\S 4 . \Sigma_{2}^{1}$-sets and the Ramsey property. In this section we start to show the relationship between Mathias- absoluteness and the Ramsey property of projective sets of reals.

It is well-known that for $\Sigma_{2}^{1}(\mathscr{B})$ and $\Sigma_{2}^{1}(\mathscr{L})$ there are characterizations with forcing absoluteness (cf. Theorem 2.3). Such a characterization exists also for 
$\Sigma_{2}^{1}(\mathscr{R})$. Although the proofs for the Baire property and the Lebesgue measurability are similar, the proof for the Ramsey property is different. This is because Mathias forcing does not have the countable chain condition. (But fortunately it has a lot of combinatorial properties.)

THEOREM 4.1. $V \models \Sigma_{2}^{1}(\mathscr{R})$ if and only if $V$ is $\Sigma_{3}^{1}$-Mathias-absolute.

Proof. First we prove that $\Sigma_{3}^{1}-M$-absoluteness implies $\Sigma_{2}^{1}(\mathscr{R})$. For this let $\Phi(x)$ be a $\Delta_{2}^{1}$-set:

$$
\Phi(x) \leftrightarrow \varphi(x) \leftrightarrow \psi(x),
$$

where $\varphi(x)$ is a $\Sigma_{2}^{1}$-set and $\psi(x)$ is a $\Pi_{2}^{1}$-set. Because $\forall x(\varphi(x) \leftrightarrow \psi(x))$ is a $\Pi_{3}^{1}$-sentence, by $\Sigma_{3}^{1}-\boldsymbol{M}$-absoluteness we have

$$
V^{M} \models \forall \tilde{x}(\varphi(\tilde{x}) \leftrightarrow \psi(\tilde{x})) .
$$

By Fact 3.9 1. we know that $V^{M} \models$ "each $\Delta_{2}^{1}$-set with parameters in $V$ is Ramsey". Therefore

$$
\boldsymbol{V}^{\boldsymbol{M}} \models \exists \tilde{y}\left(\forall \tilde{x}_{0}\left(\tilde{x}_{0} \in[\tilde{y}]^{\omega} \rightarrow \psi\left(\tilde{x}_{0}\right)\right) \vee \forall \tilde{x}_{1}\left(\tilde{x}_{1} \in[\tilde{y}]^{\omega} \rightarrow \neg \varphi\left(\tilde{x}_{1}\right)\right)\right) .
$$

But this is a $\Sigma_{3}^{1}$-sentence and because $V \models \varphi(x) \leftrightarrow \psi(x)$, also $V \models$ " $\Phi$ is Ramsey". Now because $\Phi(x)$ was arbitrary and $\Sigma_{2}^{1}(\mathscr{R})$ is equivalent to $\Delta_{2}^{1}(\mathscr{R})$ (by the Fact 3.8 5.), we have $V \models \Sigma_{2}^{!}(\mathscr{R})$.

Now we prove that $\Sigma_{2}^{1}(\mathscr{R})$ implies $\Sigma_{3}^{1}$-M-absoluteness. Let $\Psi=\exists x \psi(x)$ be a $\Sigma_{3}^{1}$-sentence. If $V \models \Psi$, then by the Shoenfield absoluteness Lemma (see [9] Theorem 98), the $\Sigma_{3}^{1}$-sentences are upwards absolute, hence $V^{M} \models \Psi$. For the other direction assume that $V^{M} \models \Psi$. Then, because of $V^{M}$ is full (cf. [9] Lemma 18.6), there is a name $\tilde{x}$, such that $V^{M} \models \psi(\tilde{x})$. By Corollary 3.10 there exist reals $r, \bar{x} \in V$ such that $\bar{x} \subseteq r$ and $V \models$ " $r \Vdash_{M} \bar{x}=\tilde{x}$ ". Now, because $V \models \Sigma_{2}^{1}(\mathscr{R})$, there is an $s \in[r]^{\omega}$ such that $s$ is $\boldsymbol{P}_{s}$-generic over $L[\bar{x}][r]\left[D^{s}\right]$. Let $m \subseteq s$ be a Mathias real over $V$, then $m$ is also $\boldsymbol{P}_{s}$-generic over $L[\bar{x}][r]\left[D^{s}\right]$ (by 3.8 2.). $V[m] \models \psi(\bar{x}[m])$, hence $L[\bar{x}][r]\left[D^{s}\right][m] \models \psi(\bar{x}[m])$ because $\psi$ is $\Pi_{2}^{1}, m \subseteq s \subseteq r$ and $\bar{x}$ may be regarded also as a $\boldsymbol{P}_{s}$-name. So there must be a condition $p \in L[\bar{x}][r]\left[D^{s}\right]$ such that $L[\bar{x}][r]\left[D^{s}\right] \models$ " $p \vdash_{P_{s}} \psi(\bar{x})$ ". Let $k=\max (\operatorname{range}(\operatorname{stem}(p)))$, then $s^{\prime}:=s \backslash k$ is $\boldsymbol{P}_{s}$-generic over $L[\bar{x}][r]\left[D^{s}\right]$ and there is an $n \in \omega$ such that $s^{\prime \prime}:=\left(s^{\prime} \backslash n\right) \cup \operatorname{stem}(p)$ satisfies $p$, (by [14] Definition 1.8 and Lemma 1.12). Hence (again by [14] Lemma 1.12), $s^{\prime \prime}$ is $\boldsymbol{P}_{s}$-generic over $L[\bar{x}][r]\left[D^{s}\right]$ and because of $s^{\prime \prime}$ satisfies $p$ and $p \vdash_{P_{s}} \psi(\bar{x})$ we have $L[\bar{x}][r]\left[D^{s}\right]\left[s^{\prime \prime}\right] \models \psi\left(\bar{x}\left[s^{\prime \prime}\right]\right)$ and finally $V \models \exists x \psi(x)$, (by Shoenfield).

So, we have found a forcing characterization for $\Sigma_{2}^{1}(\mathscr{R})$. Such a characterization with Mathias forcing does not exist for higher degrees of Mathias-absoluteness as we will show in the next section.

\section{§5. $\Sigma_{4}^{1}$-M-absoluteness and the Ramsey property.}

THEOREM 5.1. $\Sigma_{4}^{1}$-Mathias-absoluteness implies $\Delta_{3}^{1}(\mathscr{R})$.

Proof. Assume that $V$ is $\Sigma_{4}^{1}-\boldsymbol{M}$-absolute. Let $\Phi(x)$ be a $\Delta_{3}^{1}$-set in $V$ with parameters in $V: \Phi(x) \leftrightarrow \varphi(x) \leftrightarrow \psi(x)$ where $\varphi(x)$ is a $\Sigma_{3}^{1}$-set and $\psi(x)$ is a $\Pi_{3}^{1}$-set. So $V \models \forall x(\varphi(x) \leftrightarrow \psi(x))$ and $\forall x((\varphi(x) \vee \neg \psi(x)) \wedge(\neg \varphi(x) \vee \psi(x)))$ is a $\Pi_{4}^{1}$-sentence, hence $\boldsymbol{M}$-absolute. Therefore $\Phi(x)$ is still a $\Delta_{3}^{1}$-set in $V^{M}$. 
Assume $V \models$ " $\Phi(x)$ is not Ramsey". Hence $V \models \forall x \exists y_{1} y_{2}\left(y_{1} \subseteq x \wedge y_{2} \subseteq\right.$ $\left.x \wedge \Phi\left(y_{1}\right) \wedge \neg \Phi\left(y_{2}\right)\right)$. Obviously we have $\Phi\left(y_{1}\right)$ iff $\varphi\left(y_{1}\right)$ and $\neg \Phi\left(y_{2}\right)$ iff $\neg \psi\left(y_{2}\right)$ but $\varphi(x), \neg \psi(x)$ are both $\Sigma_{3}^{1}$-sets. So $V \models$ " $\Phi(x)$ is not Ramsey" is equivalent to

$$
V \models \forall x \exists y_{1} y_{2}\left(y_{1} \subseteq x \wedge y_{2} \subseteq x \wedge \varphi\left(y_{1}\right) \wedge \neg \psi\left(y_{2}\right)\right)(\equiv: \Theta)
$$

where $\Theta$ is a $\Pi_{4}^{1}$-sentence. Thus by $\Sigma_{4}^{1}-\boldsymbol{M}$-absoluteness we have

$$
V^{M} \models \Theta .
$$

Let $\mathbf{m}$ be the canonical name for a Mathias real $m$ over $V$. Then there is a condition $p$ with empty stem such that $p \vdash_{M} \varphi(\boldsymbol{m})$ or $p \vdash_{M} \neg \varphi(\boldsymbol{m})$, (see Lemmas 3.5 and 3.6 and Fact 3.81 .). Assume $p \vdash_{M} \varphi(\boldsymbol{m})$, then $p \vdash_{M} \exists \tilde{x} \varphi(\tilde{x})$ (otherwise $p \vdash_{M} \neg \psi(\boldsymbol{m})$ and $\neg \psi(\boldsymbol{m})$ is also $\left.\Sigma_{3}^{1}\right)$. Because each $y \in[m]^{\omega}$ is Mathias over $V$ and $\operatorname{stem}(p)=\langle\rangle$ we have $V[y] \models \varphi(y)$. Because $V[y] \subseteq V[m]$ and $\varphi$ is $\Sigma_{3}^{1}$, hence upwards absolute, $V[m]$ is also a model of $\varphi(y)$. So, we get

$$
p \vdash_{M} \exists \tilde{x} \forall \tilde{y}\left(\tilde{y} \in[\tilde{x}]^{\omega} \rightarrow \varphi(\tilde{y})\right) .
$$

Now because $V^{M} \models \forall \tilde{x}(\varphi(\tilde{x}) \leftrightarrow \psi(\tilde{x}))$ we finally have

$$
p \vdash_{M} \exists \tilde{x} \forall \tilde{y}\left(\tilde{y} \in[\tilde{x}]^{\omega} \rightarrow \varphi(\tilde{y}) \wedge \psi(\tilde{y})\right),
$$

but this is a contradiction to $(*)$.

THEOREM 5.2. $\Delta_{3}^{1}(\mathscr{R})$ does not imply $\Sigma_{4}^{!}$-Mathias-absoluteness.

Proof. For this it is enough to find a model $V$ in which all $\Delta_{3}^{1}$-sets are Ramsey, all $\Delta_{2}^{1}$-sets have the property of Baire and $\omega_{1}$ in this model is the same as $\omega_{1}^{L}$.

We have $V \models \Delta_{2}^{1}(\mathscr{B})$ if and only if for all reals $r$ in $V$ there is a real in $V$ which is Cohen over $L[r]$. To say this is a $\Pi_{4}^{1}$-sentence: For $s \in 2^{<\omega}$ consider $1-s$ as a binary code for a natural number $n(n>0)$ and let $\sharp n:=s,(\sharp 0:=\sharp 1=\langle\rangle)$. We write $n \preceq m$ if $\left.\sharp m\right|_{\lg (\sharp n)}=\sharp n$. Note that $\sharp n \subseteq \sharp m$ is an arithmetical statement. The sentence $\forall r \in[\omega]^{\omega} \exists c \in[\omega]^{\omega} \forall x \in[\omega]^{\omega}(c$ is a branch $\wedge(x \in L[r] \wedge x$ encodes a dense set $\rightarrow x \cap c \neq \emptyset)$ ) is a composition of the following sentences.

$c$ is a branch is $\forall n m((n \in c \wedge m \in c) \rightarrow(n \preceq m \vee m \preceq n))$, which is an arithmetical statement.

$x \in L[r]$ is a $\Sigma_{2}^{l}$-sentence with parameter $r$ (cf. [9] Theorem 97).

$x$ encode $a$ dense set is $\forall m \exists n(n \in x \wedge m \preceq n)$, which is arithmetic.

Finally $x \cap c \neq \emptyset$ is $\exists t(t \in x \wedge t \in c)$, which is arithmetic, too.

So, if $V$ is a model with the desired properties and $V$ is $\Sigma_{4}^{1}-\boldsymbol{M}$-absolute, for each real $r \in V[m]$ there is (in $V[m])$ a Cohen real $c$ over $L[r]$. If $r \in V[m]$ is a real and $c$ is a Cohen real over $L[r]$, then $L[r] \cap \omega^{\omega}$ is a strong measure zero set in $L[r][c]$ (see [5] Theorem 1.3) and hence we find in $V[m]$ a covering of $L \cap \omega^{\omega}$ with respect to the real $r$. So $L \cap \omega^{\omega}$ is a strong measure zero set belonging to $V$.

Now if $\omega_{1}^{L}=\omega_{1}^{V}$ then we get in $V[m]$ a strong measure zero set of cardinality $\omega_{1}$ with parameter in $V$, namely $L \cap \omega^{\omega}$, but this is a contradiction, (cf. [2], proof of Theorem 9.7 or cf. [10] Lemma 8.2 and recall that $\boldsymbol{M} \approx \boldsymbol{U} * \boldsymbol{P}_{\tilde{D}} \approx \boldsymbol{U} * \boldsymbol{P}(\tilde{D})$ ).

It leaves to construct a model $V$ with the desired properties. 
Proof. In [15] $\S 3$ they show, that an $\omega_{1}$ iteration of Mathias forcing starting from $L$, yields a model in which every $\Delta_{3}^{1}$-set is Ramsey and $\omega_{1}$ in this model is the same as $\omega_{1}^{L}$. (By the claim of Theorem 5.3 this is already enough.)

Now in [11] Lemma 1.18 it is proved that if we make a suitable $\omega_{1}$ iteration starting from $L$, and add alternately Kesef and Cohen reals, we get a model $V$ in which every $\Delta_{3}^{1}$-set is Ramsey, every $\Delta_{2}^{1}$-set has the Baire property and $\omega_{1}^{V}$ is the same as $\omega_{1}^{L}$.

The next theorem is in fact a consequence of the following: If $V$ is $\Sigma_{4}^{1}-M$-absolute, then $\omega_{1}^{V}$ is inaccessible in $L$.

THEOREM 5.3. $\Sigma_{4}^{1}$-Mathias-absoluteness implies $\Sigma_{3}^{1}(\mathscr{R})$.

Proof. We first give the following

Claim: If $V$ is $\Sigma_{4}^{1}-\boldsymbol{M}$-absolute, then for all reals $r \in V$ we have $\omega_{1}^{L[r]}<\omega_{1}^{V}$, (hence $\omega_{1}^{V}$ is inaccessible in $L$ ).

Now we show that this claim implies that $V \models \Sigma_{3}^{1}(\mathscr{R})$.

Lemma 5.4. If $V$ is $\Sigma_{4}^{1}-M$-absolute and $\forall r \in V\left(\omega_{1}^{L[r]}<\omega_{1}^{V}\right)$ then $V \models \Sigma_{3}^{1}(\mathscr{R})$.

Proof of The Lemma. Let $\Phi(x) \equiv \exists y \psi(x, y)$ be a $\Sigma_{3}^{1}$-set with parameter $a \in V$. If $V \models \exists z \forall x\left(x \in[z]^{(\prime)} \rightarrow \neg \Phi(x)\right)$, then the set $\Phi(x)$ is Ramsey in $V$. Therefore let us assume that $V \models \forall z \exists x\left(x \in[z]^{(j} \wedge \Phi(x)\right)(\equiv: \Theta)$. Because $\Theta$ is a $\Pi_{4}^{1}$-sentence with parameter $a$ and by $\Sigma_{4}^{1}-\boldsymbol{M}$-absoluteness we have $V^{M} \models \Theta$. Now there is a Mathias condition $p$ with empty stem, such that $p$ decides $\Phi(\boldsymbol{m})$. Because $V^{\boldsymbol{M}} \models \Theta, V^{\boldsymbol{M}}$ is a model of $\exists \tilde{x}\left(\tilde{x} \in[\mathbf{m}]^{\prime \prime \prime} \wedge \Phi(\tilde{x})\right)$. Further $V^{M}$ is full and $\Phi(x) \equiv \exists y \psi(x, y)$, hence we find Mathias names $\tilde{x}, \tilde{y}$ such that $V^{M} \models\left(\tilde{x} \in[\mathbf{m}]^{\omega} \wedge \psi(\tilde{x}, \tilde{y})\right)$.

Consider the statement $V[m] \models \exists y \psi(x, y) \Leftrightarrow V[x] \models \exists z \psi(x, z)$ and further assume that $V \models " q \vdash_{M} \psi(\tilde{x}, \tilde{y}) \wedge V[\tilde{x}] \forall \exists \tilde{z} \psi(\tilde{x}, \tilde{z})$ " (for an M-condition $q$ ). First we have to define the meaning of $q \vdash_{M}$ " $V[\tilde{x}] \models \Psi(\tilde{x})$ " where $\Psi$ is an arbitrary formula with at most one free variable: If $\tilde{z}$ is a variable in $\Psi$ for a real and $\check{n} \in \tilde{z}$ is

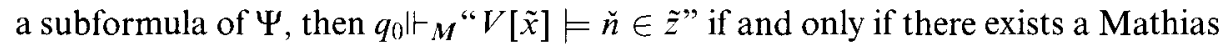
condition $\langle u, U\rangle$ such that

$$
\langle u, U\rangle \vdash_{M} \check{n} \in \tilde{z} \text { and } q_{0}{ }^{\prime}{ }_{M} \forall \breve{k}(\check{k} \in \check{u} \rightarrow \check{k} \in \tilde{x} \wedge \check{k} \in \tilde{x} \rightarrow(\check{k} \in \check{U} \vee \check{k} \in \check{u}))
$$

Let $x$ be the evaluation of $\tilde{x}$ by the Mathias real $m$. Now because $V^{M} \models \tilde{x} \subseteq \mathbf{m}, x$ is also Mathias over $V$ and $V[x] \models \Psi(x)$ if and only if there exists a Mathias condition $q_{0} \in V$ such that $q_{0} \in m$ and $q_{0} \vdash_{M} V[\tilde{x}] \models \Psi(\tilde{x})$. Thus " $q \vdash_{M} V[\tilde{x}] \models \Psi(\tilde{x})$ " is well defined.

Let $r, \bar{x}, \bar{y} \in V$ be such that $r \leq q$ and $V \models " r \vdash_{M} \bar{x}=\tilde{x} \wedge \bar{y}=\tilde{y}$." Further let $r \in$ $s \in V$ be Ramsey over $L[a][r][\bar{x}, \bar{y}]$, then there is a condition $p_{0} \in L[a][r][\bar{x}, \bar{y}]\left[D^{s}\right]$, $p_{0} \leq r$ such that $L[a][r][\bar{x}, \bar{y}]\left[D^{\prime}\right] \models$ " $p_{0} \vdash_{P_{i}} \psi(\bar{x}, \bar{y})$ ". This is because if $m^{\prime} \leq s$ is Mathias over $V$, then $m^{\prime}$ is $\boldsymbol{P}_{s}$-generic and $L[a][r][\bar{x}, \bar{y}]\left[D^{s}\right]\left[m^{\prime}\right] \models \psi\left(\bar{x}\left[m^{\prime}\right], \bar{y}\left[m^{\prime}\right]\right)$ (by Shoenfield). Let $s^{\prime}$ be $\boldsymbol{P}_{s}$-generic such that $p_{0} \in s^{\prime}$, further let $s^{\prime} \in m$ be Mathias over $V$ and $x:=\bar{x}[m](=\tilde{x}[m])$. We write $\boldsymbol{P}_{s}$ as a two step iteration $Q_{1} * \tilde{Q}_{2}$ and choose $g_{1}$ such that $g_{1}$ is $Q_{1}$-generic over $L[a][r][\bar{x}, \bar{y}]\left[D^{s}\right](=: N)$ and $N\left[g_{1}\right]=$ $N[x]$. Because of $N[x] \subseteq V[x], V[x] \cap[\omega]^{\omega} \cap N[x]$ is a $\Sigma_{2}^{1}$-set in $V[x]$ and $\forall x \exists y \forall z\left(z \in N[x] \rightarrow \exists n\left(y_{n}=z\right)\right)$ (this is: for all $x, \omega_{1}{ }^{N[x]}$ is countable) is a $\Pi_{4}^{1}$-sentence. Because of $x \subseteq m$ is Mathias over $V$ and $V$ is $\Sigma_{4}^{1}$-M-absolute, it 
follows that $\omega_{1} V[x]$ is inaccessible in $N[x]$. Hence, there exists a set $g_{2} \in V[x]$ which is $\tilde{Q}_{2}[x]$-generic over $N[x]$ such that $N[x]\left[g_{2}\right] \models \psi\left(x, \bar{y}\left[g_{1} * g_{2}\right]\right)$. Now $N[x]\left[g_{2}\right] \models \exists y \psi(x, y)$ and $N[x]\left[g_{2}\right] \subseteq V[x]$ and because $\Sigma_{3}^{1}$-formulas are upwards absolute, $V[x] \models \exists y \psi(x, y)$, which is a contradiction to $q \vdash_{M}$ " $V[\tilde{x}] \not \exists \tilde{z} \psi(\tilde{x}, \tilde{z})$ ".

(If $m$ is Mathias over $V$ and $x \in[m]^{\omega} \cap V[m]$, then we say that $V$ is $\Sigma_{n}^{1}-M$-correct if for every $\Sigma_{n}^{1}$-set $\Phi(x)$ with parameters in $V: V[m] \models \Phi(x) \Leftrightarrow V[x] \models \Phi(x)$.)

Let $p$ be a Mathias condition with empty stem which decides $\Phi(\boldsymbol{m})$, where $m$ is Mathias over $V$. Thus

$$
V \models " p \vdash_{M} \exists \tilde{z} \psi(\tilde{z}, \boldsymbol{m}) " \text { or } V \models " p \vdash_{M} \neg \Phi(\boldsymbol{m}) " .
$$

If the first case holds, let $r, \bar{z}$ be such that: $r \subseteq p$ and if $m \subseteq r$ is Mathias over $V$, then $V[m] \models \psi(\bar{z}[m], m)$. In $V$ there exists a Ramsey real $s \subseteq r$ over $L[a][r][\bar{z}]$ and because $\Pi_{2}^{1}$-sets are absolute (by Shoenfield) in $L[a][r][\bar{z}]$ there exists a $\boldsymbol{P}_{s}$-condition $q$ with empty stem (note that all $t \in[s]^{\omega}$ are also Ramsey over $L[a][r][\bar{z}]$ ) such that $L[a][r][\bar{z}]\left[D^{s}\right] \models " q \vdash_{P_{s}} \psi(\bar{z}, \boldsymbol{g})$ " where $\boldsymbol{g}$ is the canonical name for the $\boldsymbol{P}_{s}$-generic real over $L[a][r][\bar{z}]\left[D^{s}\right]$. In $V$ there is a $\boldsymbol{P}_{s}$-generic real $s^{\prime}$ such that $s^{\prime} \subseteq q$, hence for all $t \in\left[s^{\prime}\right]^{\omega}: L[a][r][\bar{z}]\left[D^{s}\right][t] \models \psi(\bar{z}[t], t)$. Again by Shoenfield we get:

$$
V \models \psi(\bar{z}[t], t) \text { and this implies } V \models \exists y \forall x \in[y]^{(\omega)} \Phi(x) \text {. }
$$

Therefore the set $\Phi(x)$ is Ramsey in $V$.

If the second case holds, we get

$$
V \models " p \vdash_{M} \forall \tilde{x} \in[\mathbf{m}]^{\omega} \neg \Phi(\tilde{x}) "
$$

hence $V \models " p \vdash_{M} \exists \tilde{y} \forall \tilde{x} \in[\tilde{y}]^{\omega} \neg \Phi(\tilde{x})$ " which is a $\Sigma_{4}^{1}$-sentence (with parameters in $V)$ and says, that $\Phi(x)$ is Ramsey. Therefore by $\Sigma_{4}^{1}-M$-absoluteness the set $\Phi(x)$ has to be Ramsey in $V$.

Now we have to show that the claim holds.

Proof of the Claim. Assume $V$ is $\Sigma_{4}^{1}-M$-absolute, then by Theorem $4.1 V \models$ $\Sigma_{2}^{1}(\mathscr{R})$, and by the Facts $3.85 ., 3.86$. and 3.92. the following is true in $V$ :

$$
\left.\forall u \in[\omega]^{\omega} \forall r \in L[u] \cap[\omega]^{\omega} \exists s \in[r]^{\omega} \text { (sis Ramsey over } L[u]\left[D^{s}\right]\right) .
$$

To say this is a $\Pi_{4}^{1}$-sentence:

$$
\text { Define } b: \begin{aligned}
{[\omega]^{2} } & \longrightarrow \omega \\
\{n, m\} & \longmapsto \frac{1}{2}\left(\max (\{n, m\})^{2}-\max (\{n, m\})\right)+\min (\{n, m\}) .
\end{aligned}
$$

Note that $b$ is a bijection and arithmetic. With $b$ we can consider each $\pi \in[\omega]^{\omega}$ as a function from $[\omega]^{2}$ to 2 , namely by

$$
\pi(\{n, m\})=0 \Longleftrightarrow b\{n, m\} \in \pi .
$$

The sentence

$$
\forall u \in[\omega]^{\omega} \forall r \in L[u] \cap[\omega]^{\omega} \exists s \in[r]^{\omega}\left(s \text { is } \boldsymbol{P}_{s} \text {-generic over } L[u]\left[D^{s}\right]\right)
$$

is a composition of the following sentences.

$r \in L[u]$ is a $\Sigma_{2}^{1}$-sentence with parameter $u$.

$s \in[r]^{\omega}$ is $\forall i(i \in s \rightarrow i \in r)$, which is arithmetic. 
$s$ is $\boldsymbol{P}_{s}$-generic over $L[u]\left[D^{s}\right]$, which is again a composition of the following sentences.

$x \in L[u]\left[D^{s}\right]$ is a $\Sigma_{2}^{1}$-sentence with parameters $u$ and $s$.

$\left.\pi\right|_{[s \backslash n]^{2}}$ is constant is an arithmetical sentence because of $b$ is arithmetic.

$D^{s}$ is an ultrafilter in $L[u]\left[D^{s}\right]$ is $\forall x \in L[u]\left[D^{s}\right] \cap[\omega]^{\omega} \exists n(s \backslash n \subseteq x \vee s \backslash n \cap x=\emptyset)$, which is a $\Pi_{2}^{1}$-sentence with parameters $u$ and $s$.

$\forall \pi \in L[u]\left[D^{s}\right] \exists n\left(\left.\pi\right|_{[s \backslash n]^{2}}\right.$ is constant), which is also a $\Pi_{2}^{1}$-sentence with the parameters $u$ and $s$.

Therefore if $V$ is $\Sigma_{4}^{1}-M$-absolute, in $V^{M}$ for each real $u$ there exists a real $s$ which dominates the reals of $L[u$ ] (cf. Fact 3.92 .). Let $m$ be Mathias over $V$. Because $\boldsymbol{M}$ is flexible (cf. Lemma 3.7), $\boldsymbol{M}$ adds a dominating family of size $\omega_{1}$ (see [3] Theorem 3.1). If there is a real $r \in V$ such that $\omega_{1}^{L[r]}=\omega_{1}^{V}$ and $m$ is Mathias over $V$, then the reals of $L[r][m]$ dominates the reals of $V[m]$. (Note that the $\boldsymbol{M}$-names $\breve{f}_{\alpha}\left(\alpha<\omega_{1}\right)$ which are constructed in [3] Theorem 3.1 can all be defined within $L[r]$.) But this contradicts that in $V[m]$ we have a dominating real over $L[r][m]$.

This concludes the proof of the Theorem.

We can prove even more, as we will see in the next section.

\section{§6. Higher degrees of Mathias-absoluteness.}

Corollary 6.1. $\Sigma_{5}^{1}$-Mathias-absoluteness implies $\Delta_{4}^{1}(\mathscr{R})$.

Proof. Let $\Phi(x)$ be a $\Delta_{4}^{1}$-set:

$$
\Phi(x) \leftrightarrow \varphi(x) \leftrightarrow \neg \psi(x)
$$

where $\varphi(x)$ and $\psi(x)$ are $\Sigma_{4}^{1}$-sets. By $\Sigma_{5}^{1}$ - $M$-absoluteness, $\Phi(x)$ is still a $\Delta_{4}^{1}$-set in $V^{M}$. Let $p$ be an $\boldsymbol{M}$-condition with empty stem such that

$$
V \models " p \vdash_{M} \varphi(\mathbf{m}) ",
$$

(if $V \models " p \Vdash_{M} \neg \varphi(\mathbf{m})$ " then $V \models " p \Vdash_{M} \psi(\mathbf{m})$ "), then there is an $\boldsymbol{M}$-name $\tilde{y}$ and (by Corollary 3.11) a $p^{\prime} \subseteq p$ with empty stem, such that

$$
V \models " p^{\prime} \Vdash_{M} \varphi_{0}(\mathbf{m}, \tilde{y}) "
$$

(where $\varphi(x) \equiv \exists y \varphi(x, y)$ and $\varphi_{0}$ is a $\Pi_{3}^{1}$-formula). Let $m \subseteq p^{\prime}$ be Mathias over $V$, then

$$
V[m] \models \varphi_{0}(m, \tilde{y}[m]) .
$$

Now in the proof of Lemma 5.4 in fact we showed, that if $m$ is Mathias over $V$, $m^{\prime} \in[m]^{(\prime)} \cap V[m], \forall r \in[\omega]^{(\prime)} \cap V\left(\omega_{1}^{L[r]}<\omega_{1}^{V}\right)$ and $\Phi(x)$ is a $\Sigma_{3}^{1}$-set (or a $\Pi_{3}^{1}$-set) with parameters in $V$, then

$$
V\left[m^{\prime}\right] \models \Phi\left(m^{\prime}\right) \Leftrightarrow V[m] \models \Phi\left(m^{\prime}\right) .
$$

Because of $m^{\prime} \in[m]^{(\prime)} \cap V[m], m^{\prime}$ is also Mathias over $V$ and the sentence $\forall x \in$ $[m]^{\omega}\left(\varphi_{0}(x, \tilde{y}[m])\right)$ holds in $V[m]$. Therefore $\exists z \forall x \in[z]^{\omega} \neg \psi(x)$, which is a $\Sigma_{5}^{1}$ sentence with parameters in $V$, is true in $V[m]$. Hence, $V \models$ " $\Phi(x)$ is Ramsey" and because $\Phi(x)$ was arbitrary we get $V \models \Delta_{4}^{1}(\mathscr{R})$.

To prove the last results, we need two slightly technical lemmas. 
LEMMA 6.2. If $\forall r \in[\omega]^{\omega} \cap V\left(\omega_{1}^{L[r]}<\omega_{1}^{V}\right)$ and $\Phi(\tilde{z})$ is a $\Sigma_{3}^{1}$-formula (where $\tilde{z}$ is an $\boldsymbol{M}$-name in $V$ for a real), then: for all $\boldsymbol{M}$-conditions $q$ in $V$ there is a real $a$ and an $\boldsymbol{M}$-condition $l$ in $V$ such that ( $q$ is an $\boldsymbol{M}$-condition in $L[a]$ and $l \leq q$ ) and for all reals $m$ : if $m$ is Mathias over $V$ and $l \in m$, then ( $m$ is Mathias over $L[a]$ and $\tilde{z}[m] \in L[a]$ and $(L[a][m] \models \Phi(\tilde{z}[m])$ if and only if $V[m] \models \Phi(\tilde{z}[m])))$.

Proof. To simplify the notation we assume that the parameters of $\Phi$ are in $L$. Assume $V \models " q_{0} \Vdash_{M} \Psi(\tilde{z}, \tilde{x})$ " where $q_{0} \leq q$ and $\Phi(z) \equiv \exists x \Psi(z, x)$. Let $r_{0} \leq q_{0}$ and $\bar{z}, \bar{x}$ such that $V \models " r_{0} \vdash_{M} \bar{z}=\tilde{z} \wedge \bar{x}=\tilde{x}$ ". Let $a$ be a real which encode the reals $r_{0}, \bar{z}, \bar{x}$ and $q$. In $L[a]$ there must be an $\boldsymbol{M}$-condition $q_{1} \leq r_{0}$ such that $L[a] \models " q_{1} \Vdash_{M} \Psi(\bar{z}, \bar{x})$ " (because of the absoluteness of $\Pi_{2}^{1}$-formulas). Let $l \in V$ be Mathias over $L[a]$ such that $q_{1} \in l$ and further let $m$ be Mathias over $V$ such that $l \in m$, then $L[a][m] \models \Psi(\tilde{z}[m], \tilde{x}[m])$ and $V[a][m] \models \Psi(\tilde{z}[m], \tilde{x}[m])$.

If $V \models " q_{0} \Vdash_{M} \neg \Phi(\tilde{z})$ " for all $q_{0} \leq q$ which decides $\Phi(\tilde{z})$, there is an $\boldsymbol{M}$ condition $q_{1}$ as in the former case, (because $\Pi_{3}^{1}$-formulas are downwards absolute). The rest of the proof in this case is the same as above.

We say $L[a]$ computes well the $\Sigma_{3}^{1}$ formula $\Phi(\tilde{z})$ (the $\Pi_{3}^{1}$ formula $\neg \Phi(\tilde{z})$, respectively) with respect to $q_{1}$.

LeMMA 6.3. If $V$ is $\Sigma_{4}^{1}-\boldsymbol{M}$-absolute, then $V$ is $\Sigma_{4}^{1}-\boldsymbol{M}$-correct.

Proof. If not, then there is a $\Sigma_{4}^{1}$-formula $\Phi(x)$ and an $\boldsymbol{M}$-condition $p \in V$ such that $V \models " p \Vdash_{M} \tilde{x} \in[\boldsymbol{m}]^{\omega} \wedge \Phi(\tilde{x}) \wedge V[\tilde{x}] \not \models \Phi(\tilde{x}) "$. Because $V \models " p \Vdash_{M} \Phi(\tilde{x}) "$ there is an $M$-name $\tilde{y}$ such that $V \models " p \vdash_{M} \Psi(\tilde{x}, \tilde{y}) "$ where $\Phi(x) \equiv \exists y \Psi(x, y)$ and $\Psi(x, y)$ is a $\Pi_{3}^{1}$-formula.

Let $r, \bar{x}, \bar{y}$ be such that $r \leq p$ and $V \models " r \Vdash_{M} \tilde{x}=\bar{x} \wedge \tilde{y}=\bar{y}$." By Lemma 6.2 there is an $a \in V$ and an $M$-condition $q \leq r$ such that $L[a]$ computes well $\Psi(\bar{x}, \bar{y})$ with respect to $q$. Let $l$ and $m$ as in the Lemma 6.2 and further let $x:=\bar{x}[m]$. Because $m$ is Mathias over $L[a]$ and $x \in L[a][m]$ we can write the Mathias forcing as a two step iteration $Q_{1} * \tilde{Q}_{2}$ and choose (as in the proof of Lemma 5.4) $g_{1}, g_{2} \in V[x]$ such that $g_{1}$ is $Q_{1}$-generic over $L[a], g_{2}$ is $\tilde{Q}_{2}\left[g_{1}\right]$-generic over $L[a]\left[g_{1}\right], g_{1} * g_{2}$ is $\boldsymbol{M}$-generic over $L[a]$ with respect to $q$ and $L[a]\left[g_{1}\right]=L[a][x]$. With the same arguments as in the proof of Lemma 5.4 we have $L[a][x]\left[g_{2}\right] \models \Psi\left(x, \bar{y}\left[g_{1} * g_{2}\right]\right)$. Now because $L[a]$ computes well the $\Pi_{3}^{1}$-formula $\Psi$ and $g_{2} \in V[x]$, we finally have $V[x] \vDash=\Phi(x)$.

THEOREM 6.4. $\Sigma_{6}^{1}$-Mathias-absoluteness implies $\Sigma_{4}^{1}(\mathscr{R})$.

ProOF. Let $\Phi(x)$ be a $\Sigma_{4}^{1}$-formula with parameters in $V$ and further let $p \in V$ be an $\boldsymbol{M}$-condition which decides $\boldsymbol{\Phi}(\boldsymbol{m})$.

If $V \models " p \Vdash_{M} \Phi(\boldsymbol{m})$ " then by Lemma $6.3 V \models$ " $p \Vdash_{M} \exists x \forall y \in[x]^{(\omega)} \Phi(y)$ ".

If $V \models " p \vdash_{M} \neg \Phi(\boldsymbol{m})$ " then by Lemma $6.3 V \models " p \vdash_{M} \exists x \forall y \in[x]^{(")} \neg \Phi(y)$ ".

In both cases (by $\Sigma_{6}^{1}-M$-absoluteness) we get that $\Phi(x)$ is Ramsey in $V$ and because $\Phi(x)$ was arbitrary we have $V \models \Sigma_{4}^{1}(\mathscr{R})$.

COROLlaRY 6.5. $\Sigma_{6}^{1}$-Mathias-absoluteness implies $\Delta_{5}^{1}(\mathscr{R})$.

Proof. Let $\Phi(x)$ be a $\Delta_{5}^{1}$-set:

$$
\Phi(x) \leftrightarrow \varphi(x) \leftrightarrow \neg \psi(x)
$$


where $\varphi(x)$ and $\psi(x)$ are $\Sigma_{5}^{1}$-sets. By $\Sigma_{6}^{1}-\boldsymbol{M}$-absoluteness, $\Phi(x)$ is still a $\Delta_{5}^{1}$-set in $V^{M}$. Let $p$ be an $\boldsymbol{M}$-condition with empty stem such that $V \models " p \vdash_{M} \varphi(\mathbf{m})$ ", (if $V \models " p \Vdash_{M} \neg \varphi(\mathbf{m})$ " then $V \models " p \Vdash_{M} \psi(\mathbf{m})$ "), then there is an $M$-name $\tilde{y}$ and (by Corollary 3.11 ) a $p^{\prime} \subseteq p$ with empty stem, such that

$$
V \models " p^{\prime} \Vdash_{M} \varphi_{0}(\mathbf{m}, \tilde{y}) "
$$

(where $\varphi(x) \equiv \exists y \varphi_{0}(x, y)$ and $\varphi_{0}$ is a $\Pi_{4}^{1}$-formula). Let $m \subseteq p^{\prime}$ be Mathias over $V$, then

$$
V[m] \models \varphi_{0}(m, \tilde{y}[m]) .
$$

Because of Lemma 6.3 and because $m^{\prime} \in[m]^{\omega} \cap V[m]$ is Mathias over $V$, the sentence $\forall m^{\prime} \in[m]^{\prime \prime \prime} \varphi_{0}\left(m^{\prime}, \tilde{y}\left[m^{\prime}\right]\right)$ which is $V[m] \models \exists z \forall x \in[z]^{\omega} \varphi(x)$, holds in $V[m]$. Therefore $\exists z \forall x \in[z]^{\omega} \neg \psi(x)$ which is a $\Sigma_{6}^{1}$-sentence with parameters in $V$ is true in $V[m]$.

Hence, $V \models$ " $\Phi(x)$ is Ramsey" and because $\Phi(x)$ was arbitrary we get

$$
V \models \Delta_{5}^{1}(\mathscr{R}) \text {. }
$$

\section{REFERENCES}

[1] T. BARTOSZYŃSKI and H. JUDAH, Set theory: the structure of the real line.

[2] J. E. BAUMGARTNER, Iterated forcing, Surveys in set theory (A. R. D. Mathias, editor), Cambridge University Press, London and New York, 1983, pp. 1--59.

[3] J. BRENDLE, Combinatorial properties of classical forcing notions, Annals of Pure and Applied Logic, vol. 73 (1995), pp. 143-170.

[4] J. Brown, The Ramsey sets and related $\sigma$-algebras and ideals, Fundamenta Mathematica, vol. 136 (1990), pp. 179-185.

[5] P. Corazza, Ramsey sets, the Ramsey ideal, and other classes over $\boldsymbol{R}$, this Journal, vol. 57 (1992), pp. 144I-1468. 165 .

[6] E. Ellentuck, A new proof that analytic sets are Ramsey, this Journal, vol. 39 (1974), pp. $163-$

[7] P. ERdös and R. RADo, Combinatorial theorems on classifications of subsets of a given set, Proceedings of the London Mathematical Society, vol. 2 (1952), pp. 417-439.

[8] M. Goldstern, Tools for your forcing construction, Set theory of the reals (H. Judah, editor), Israel Mathematical Conference Proceedings, Bar-Ilan University, 1993, pp. 305-360.

[9] TH. JeCH, Set theory, Academic Press, London, 1978.

[10] - Multiple forcing, Cambridge University Press, Cambridge, 1987.

[11] H. JUDAH, Exact equiconsistency results for $\Delta_{3}^{1}$-sets of reals, Archive for Mathematical Logic, vol. 32 (1992), pp. 101-112.

[12] - - , $\Delta_{3}^{1}$-sets of reals, Set theory of the reals, Israel Mathematical Conference Proceedings, Bar-Ilan University, 1993, pp. 361-384.

[13] — , Absoluteness for projective sets, Logic colloquium '90 (J. Oikkonen et al., editors), Springer-Verlag, Berlin, 1993, pp. 145-154.

[14] H. Judah and S. Shelah, $\Delta_{2}^{1}$-sets of reals, Annals of Pure and Applied Logic, vol. 42 (1989), pp. 207-223.

$[15] \longrightarrow, \Delta_{3}^{1}$-sets of reals, this JoURNAL, vol. 58 (1993), pp. 72-80.

[16] K. KUNEN, Set theory, an introduction to independence proofs, North-Holland, Amsterdam, 1983.

[17] A. R. D. Mathias, Happy families, Annals of Mathematical Logic, vol. 12 (1977), pp. 59-111.

[18] J. Silver, Every analytic set is Ramsey, this Journal, vol. 35 (1970), pp. 60-64. 
DEPARTEMENT MATHEMATIK

ETH ZENTRUM

8092 ZÜRICH, SWITZERLAND

E-mail: halbeis@math.ethz.ch

DEPARTMENT OF MATHEMATICS

BAR-ILAN UNIVERSITY

52900 RAMAT GAN, ISRAEL

E-mail: judah@bimacs.cs.biu.ac.il 